\title{
Síndrome de Ekbom no sudoeste da Amazônia brasileira: relato de caso
}

\author{
Ekbom syndrome in the southwest of the Brazilian Amazon: report of case
}

Síndrome de Ekbom en el suroeste de la Amazonia brasileña: informe de caso

Lísia Ferreira Gonçalves ${ }^{1 *}$, Caroline Linhares Ferreira Craveiro ${ }^{1}$, Giovana Maria Leite de Souza ${ }^{1}$, Isis Marinho França ${ }^{1}$, Louise Araújo Lambert ${ }^{1}$, Paulo Artur da Silva Rodrigues ${ }^{1}$, Roberto Egídio Brelaz Goulart $^{1}$, Suellen Gleyce França da Silva², Rita de Cássia Ribeiro Pereira² ${ }^{2}$ Camila Costa Lima².

\section{RESUMO}

Objetivo: Relatar um caso de rara ocorrência, etiopatogenia desconhecida, maior prevalência em idosos e associação com outros distúrbios psiquiátricos no estado do Acre. Detalhamento do caso: A síndrome de Ekbom (SE), ou delírio parasitário, é um estado delirante de caráter fóbico obsessivo, em que o paciente imagina e acredita que está infectado por parasitas, que saem da pele. Esta crença faz com que o paciente cause lesões em si mesmo. O caso apresentado é ilustrativo da Síndrome de Ekbom e aproxima-se muito do protótipo descrito na literatura. Considerações finais: Por se tratar de uma condição rara, é necessário ser investigado minuciosamente para excluir outras possíveis causas de delirium e/ou demências em pacientes com quadros semelhantes. Será possível observar uma resistência à adesão ao tratamento pelo paciente. Logo, o atendimento em conjunto com a psiquiatria deve ser instituído. O tratamento da SE é realizado através do uso de antipsicótico, sendo Pimozida o fármaco de primeira escolha.

Palavras-chave: Dermatologia, Prurido, Delírio de parasitose, Pimazida.

\begin{abstract}
Objective: To report one case of rare occurrence, unknown etiopathogenesis, higher prevalence in the elderly and association with other psychiatric disorders in the state of Acre. Case details: Ekbom's syndrome (SE), or parasitic delirium, is a delusional state of obsessive phobic character, in which the patient imagines and believes that he is infected by parasites, which leave the skin. This belief causes the patient to injure himself. The case presented is illustrative of Ekbom's Syndrome and are very close to the prototype described in the literature. Final considerations: As it is a rare condition, it is necessary to be thoroughly investigated to exclude other possible causes of delirium and / or dementia in patients with similar conditions. It will be possible to observe resistance to treatment adherence by the patient. Therefore, joint care with psychiatry should be instituted. The treatment of SE is performed through the use of antipsychotic drugs, with Pimozide being the drug of choice.
\end{abstract}

Key words: Dermatology, Pruritus, Delusional parasitosis, Pimazide.

\section{RESUMEN}

Objetivo: Reportar uno caso de ocurrencia rara, etiopatogenia desconocida, mayor prevalencia en ancianos y asociación con otros trastornos psiquiátricos en el estado de Acre. Detalles del caso: El síndrome de Ekbom (SE), o delirio parasitario, es un estado delirante de carácter obsesivo fóbico, en el que el paciente imagina y cree que está infectado por parásitos, que salen de la piel. Esta creencia hace que el paciente se lastime. Los casos presentados son ilustrativos del Síndrome de Ekbom y se acercan mucho al prototipo

${ }^{1}$ Centro Universitário UNINORTE, Rio Branco - AC. *E-mail: lisiafg8@gmail.com

2 Universidade Federal do Acre (UFAC), Rio Branco - AC. 
descrito en la literatura. Consideraciones finales: Como es una condición poco común, es necesario investigarlo a fondo para excluir otras posibles causas de delirio y / o demencia en pacientes con condiciones similares. Será posible observar resistencia a la adherencia al tratamiento por parte del paciente. Por lo tanto, debe instituirse la atención conjunta con la psiquiatría. El tratamiento de la SE se realiza mediante el uso de fármacos antipsicóticos, siendo la Pimozida el fármaco de elección.

Palabras clave: Dermatología, Prurito, Delirio de parasitosis, Pimazida.

\section{INTRODUÇÃO}

A Síndrome de Ekbom (SE), parasitose delirante ou delírio parasitário, se caracteriza por um estado insidioso e delirante de caráter fóbico-obsessivo, em que o paciente acredita estar infectado por parasitas, que saem da pele- em geral, couro cabeludo, olhos, boca ou região genital (VIDAL CEL, et al., 2009).

Alguns pacientes, em estado alucinatório, veem os parasitas e retiram fragmentos da pele, causando lesões e automutilações. Outrora, coletam cabelo, tecido descamativo ou outras substâncias, as quais levam em saco plástico ao médico, solicitando que haja avaliação laboratorial, conhecido na medicina como o "sinal da caixa de fósforos", patognomônico da SE (GOI PD e SCHARLAU CT, 2007).

Esta crença de parasitas saindo da pele pode fazer com que outros indivíduos, que moram juntos, passem a apresentar os mesmos sintomas, gerando, assim, o transtorno delirante induzido, fenômeno conhecido como folie à deux (ARCE EPD, et al., 2017; JUNIOR QC e CORBETT CEP, 2003).

Nos pacientes com SE, as lesões cutâneas são frequentes, apresentando-se como escoriações discretas, úlceras francas ou cicatrizes, produzidas pelo paciente no ato de tentativa de "retirada" do parasita da pele. A maioria dos pacientes descrevem com detalhes a morfologia, o ciclo vital e os hábitos desses parasitas, assim como referem a forma como agem para livrar-se deles (KOO J e LEE CS, 2001).

Historicamente, os primeiros casos da SE foram descritos na literatura no final do século XIX, por volta de 1894, por Thirbierge, na França, como acarophobia (acarofobia) e chamado por Perrin, em 1896, como nevrodermie parasitophobique primitive (neurodermia parasitofóbica). Mais tarde, em 1938, na Suíça, um médico chamado Ekbom descreveu sete casos e os denominou de dermatozoenwahn (delírio dermatozóico), o qual acreditava que alterações das sensações, como parestesia, levavam o indivíduo a crer que estava infestado por parasitas. Até os dias de hoje ainda não está claro a etiopatogenia da síndrome em questão (JUNIOR QC e CORBETT CEP, 2003).

Por se enquadrar em uma síndrome rara, a Parasitose Delirante possui prevalência de 83,21 casos para cada milhão de habitantes. Afeta majoritariamente mulheres (incidência 3:1 em relação aos homens), e principalmente indivíduos acima de 50 anos, com idade média de início da doença por volta dos 55 anos (ALVES CJM, et al., 2010). Trata-se de casos subnotificados, em que, a maioria das vezes, o paciente se nega a aceitar o diagnóstico psiquiátrico e passa a procurar outros profissionais da saúde. O dermatologista geralmente é o primeiro especialista a ser procurado por este paciente, porém, estima-se que um dermatologista experiente se depararia com o quadro de um paciente com esta infestação ilusória a cada sete anos de prática (AMANCIO EJ, et al., 2002).

Por se tratar de uma doença desconhecida por muitos profissionais, cujo diagnóstico é eminentemente clínico e de exclusão, logo, observa-se a importância de se apresentar casos desta entidade. Assim, o estudo tem como objetivo relatar um caso clínico sugestivo de Síndrome de Ekbom.

\section{DETALHAMENTO DE CASO}

Paciente feminina, 60 anos, branca, analfabeta, dona de casa, natural e residente em uma cidade no interior do Acre, deu entrada no hospital estadual com a queixa de lesão pruriginosa em região abdominal há cerca de dois anos. Relata que sentia "bichos mordendo" e consequentemente, apresentava descamação esbranquiçada na roupa. Referia que os bichos saiam do couro cabeludo e espalhavam-se pelo corpo. 
Familiar que a acompanhava, relatava ter os mesmos sintomas. Conta que, devido ao quadro, havia descartado o colchão, peças de roupas e o sofá da própria casa. Refere diagnóstico de Depressão e Transtorno Afetivo Bipolar em uso de Amitriptilina 10mg e Carbamazepina 100mg. Negava Diabetes Mellitus e referia ser hipertensa em uso de Anlodipino $5 \mathrm{mg}$ e Enalapril $5 \mathrm{mg}$. Negava qualquer uso de droga lícita ou ilícita. Refere histórico de passagem por outros profissionais, inclusive três dermatologistas anteriores, e várias tentativas de tratamentos com antibiótico, antifúngico, anti-histamínico, anti-helmíntico e corticoide, não havendo melhora.

À consulta, trouxe exames: ácaros negativo, IgE aumentado, com valor de 261 (valor de referência: 214), entre outros, dentro da normalidade. Ao exame físico, paciente em bom estado geral, lúcida e orientada em tempo e espaço, autopsíquica e alopsíquica, vigil, eutímica, fala conexa, Glasgow 15, acianótica, anictérica, afebril, normocorada, normocárdica e eupneica. Aparelho respiratório e cardiovascular sem anormalidades. Abdome semi-globoso, normotenso, ruídos hidroaéreos presentes, timpânico à percussão, indolor à palpação superficial e profunda, com a presença de cicatrizes hipocrômicas e xerose associada aos múltiplos tratamentos prévios, além do prurido momentâneo (Figura 1).

A paciente insistia em demonstrar a presença de "insetos" sob a sua pele, na qual a mesmo, durante a avaliação, beliscava a própria pele tentando comprovar sua descrição, não sendo possível comprovar. Paciente sem outras alterações ao exame físico.

Solicitado exames laboratoriais e eletrocardiograma para descartar outras possíveis causas, foi prescrito Pimozida (Orap) 1mg/dia durante duas semanas, sendo após este período, dose aumentada para $2 \mathrm{mg} / \mathrm{dia}$. Além de suspender a Carbamazepina, foi solicitado avaliação psicológica e encaminhamento ao psiquiatra.

No retorno, após quatro meses, exames dentro da normalidade, paciente apresentava persistência do quadro, referindo não ter utilizado medicações de forma adequada por queixas de mal-estar e náuseas. Realizado nova prescrição após recomendação de um psiquiatra, iniciou-se Olanzapina 2,5mg, além do encaminhamento para psiquiatria. Paciente não apresentou melhora do quadro pela não adesão ao tratamento e não aceitação diagnóstica.
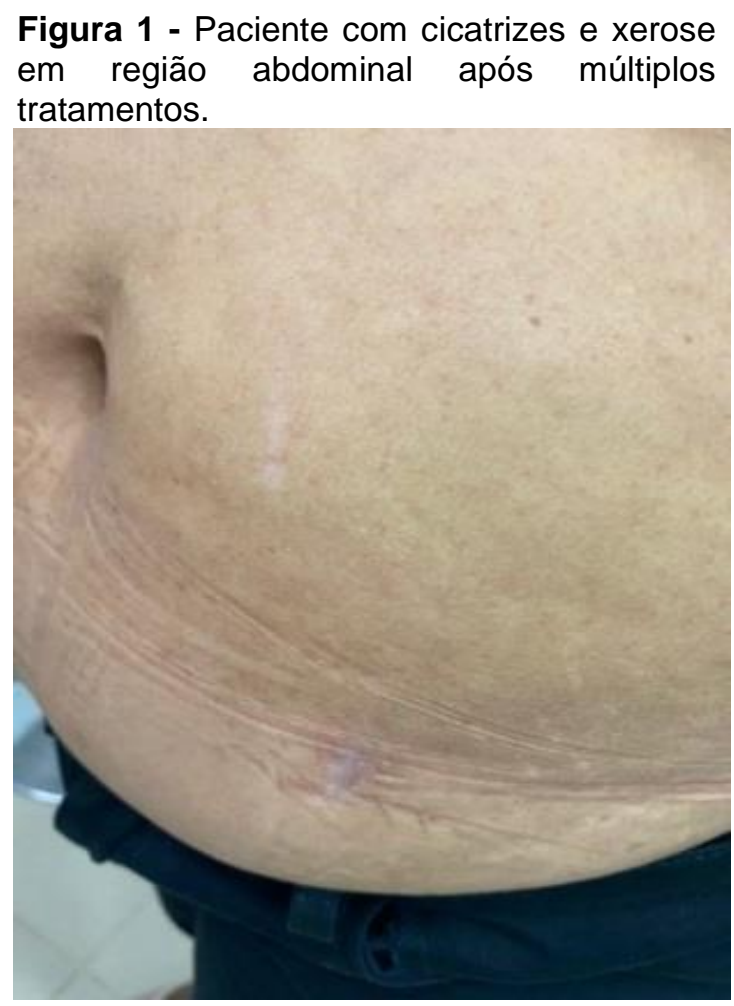

Fonte: Gonçalves LF, et al., 2020. 


\section{DISCUSSÃO}

O caso apresentado é ilustrativo da Síndrome de Ekbom, aproxima-se muito do protótipo descrito na literatura, cujos critérios são pacientes do sexo feminino, já idosas ou em período pré-senil, em situação de isolamento social. A paciente em questão se enquadra nos seguintes critérios: faixa etária, ficando na média dos 55 anos, sexo e nível de escolaridade baixa (ALVES CJM, et al., 2010).

Como se observa no caso, por não adesão adequada ao tratamento e não aceitação diagnóstica, não foi possível observar um desfecho favorável. É importante ressaltar que houve várias tentativas de tratamento prévio, de variadas possíveis causas e, todas, sem resposta adequada. Segundo Amâncio et al. (2002), a maioria dos quadros de SE se encaixam nas seguintes características: ser de ocorrência rara, ter envolvimento do couro cabeludo, acometer principalmente mulheres de meia-idade ou idosas, além do fato de ser de natureza crônica e pouco responsiva ao tratamento com antipsicóticos (exceto Pimozida).

Além do mais, neste caso, houve a presença do fenômeno conhecido como folie à deux, no qual o familiar relatava os mesmos sintomas. Miller L (1954) há anos destacava que $10-15 \%$ dos casos apresentavam este fenômeno do transtorno delirante induzido, também podendo surgir como folie a trois, ou mesmo, afetar uma família inteira. Há quem considere a SE como a mais contagiosa das síndromes psiquiátricas (MARQUES JG, 2008).

O diagnóstico do delírio da SE é clínico, mas é importante considerar a existência de outras doenças ou distúrbios associados, como exemplos, a escabiose, a foliculite crônica, a reação alérgica a picada de insetos, as intoxicações medicamentosas, entre outros. Além disso, excluir possíveis causas hematológicas, endócrinas, neurológicas, infecciosas, entre outras, que poderiam estar gerando um quadro clínico semelhante. Devem-se associar também a outros distúrbios que levem a alterações da sensibilidade, quadros de demências, de neoplasias, de deficiência de vitaminas (em especial B12), de doenças cerebrovasculares ou pacientes em uso de substâncias (geralmente cocaína) ou medicamentos. Alguns desses distúrbios que levam a sintomas cutâneos, como pruridos, podem contribuir com o início do delírio (ALVES CJM, et al., 2010; MARQUES JG, 2008; VIDAL CEL, et al., 2009).

Como a paciente em estudo queixava-se de lesões pruriginosas, apesar da sua difícil abordagem devido à inespecificidade, o prurido é um sintoma frequente nas consultas de Atenção Básica. Portanto, é necessário, mais uma vez, enfatizar a importância de uma história clínica abrangente e detalhada, destacando a região, periodicidade, intensidade, número, características das lesões e possíveis fatores precipitantes e fatores de melhora. A percepção do paciente de estar infestado por parasitas é o que chama a atenção no caso clínico em estudo. Frente a esses sintomas, devem-se considerar outras patologias psiquiátricas, dermatológicas ou neurológicas no diagnóstico diferencial (TERCERO EG, et al., 2020).

Caracterizada como um subtipo de transtorno delirante do tipo somático, a Síndrome de Ekbom, pode ser conhecida como Parasitose Delirante Primária, apesar de 60\% dos casos serem associados a outros transtornos psiquiátricos, como transtorno depressivo, bipolar, do tipo paranoia, esquizofrênico e/ou abuso ou abstinência de drogas lícitas ou ilícitas (VARGAS CD, et al., 2020). Classifica-se no Manual Diagnóstico e Estatístico de Transtornos Mentais (DSM-5) em transtornos delirantes do tipo somático $(297,1)$ e, na Classificação Internacional de Doenças (CID10), encontra-se nos transtornos delirantes (F22.0) (MUNOZ H e BAYONA L, 2015).

Outra classificação desta entidade está baseada na localização do patógeno, sendo dividida de três maneiras: endoparasitária (parasitas são sentidos dentro do corpo), ectoparasitária (os parasitas são sentidos pela pele) e mista (TERCERO EG, et al., 2020). Por sua vez, Freudenmann RW e Lepping P (2009), proponhem outra classificação que se baseia na fisiopatogenia, dividindo-se em dois tipos: primária, quando é independente de outras causas; e secundária, quando é devido à outra patologia médica.

É importante ressaltar que, embora o delírio de infestação parasitária seja reconhecido como um transtorno mental, os pacientes comumente procuram ajuda de um dermatologista ou clínico geral, e na maioria dos casos, rejeitam o encaminhamento para um psiquiatra. Como o diagnóstico é de exclusão, por isso se faz necessário um atendimento conjunto, entre o serviço da dermatologia e da psiquiatra, visto que 
não há consenso segundo qual a forma de tratamento mais apropriada (MARQUES JG, 2008; VIDAL CEL, et al., 2009).

Outro importante destaque deste caso, em relação às demais psicoses, é que a estratégia de conversação e persuasão podem não ser eficientes e o paciente acaba por não conseguir se desfazer do delírio, da alucinação, ou mesmo, de ambos (AMERICAN PSYCHIATRIC ASSOCIATION, 2013). Ademais, pode-se observar que há uma dificuldade na relação médico-paciente diante casos de Síndrome de Ekbom. Estudos destacam a alta taxa de tratamentos antiparasitários nos pacientes com transtorno de parasitose, logo, demonstrando a dificuldade existente no reconhecimento e no convencimento diagnóstico do paciente (ARCE EPD, et al., 2017; LLANOS FC, et al., 2016).

O Delírio de parasitose por ser considerado um diagnóstico raro e de exclusão, o torna desafiador, já que outras causas comuns de prurido devem ser afastadas. Porém, mais desafiador é o tratamento, podendo ser este realizado com o uso de antipsicótico, sendo Pimozida o fármaco de primeira escolha, por este bloquear os receptores opióides, assim, diminuir a sensação de prurido. Em Alves CJM, et al. (2010) observou-se que $60-80 \%$ dos pacientes respondem de maneira positiva ao tratamento com Pimozida, com melhora dos sintomas nas primeiras duas semanas, porém faz-se necessário o tratamento durante meses para a remissão completa. Por ser cardiotóxico, exige acompanhamento eletrocardiográfico antes do início e, durante o tratamento, já que a Pimozida prolonga o intervalo QT (ALVES CJM, et al., 2010).

Deve-se evitar o uso de Pimozida em qualquer paciente com alterações ou doenças cardíacas, nestes casos, sugere-se o uso de Risperidona. Por outro lado, a remissão espontânea gira em torno de $20 \%$ e, quando não tratada, pode gerar sequelas físicas e psicossociais. O maior desafio terapêutico é a adesão pelo paciente delirante ao tratamento medicamentoso (MARQUES JG, 2008).

Lepping $P$, et al. (2015) recomenda a necessidade de excluir primeiramente infestações reais com passagem por dermatologista ou especialista em doenças infecciosas, exames e testes apropriados. Além de ter sabedoria e levar em conta a angústia do paciente, sem reforçar falsas crenças. Ao ver o paciente pela primeira vez, é fundamental compreender que muito provavelmente não se conseguirá convencê-lo de que não possui animais ou patógenos em sua pele.

E, de forma primordial, faz-se necessário criar um vínculo de confiança médico-paciente para melhorar a adesão ao tratamento e alívio dos sintomas. O objetivo é ouvir os desconfortos, as frustrações e as emoções do paciente em relação à sua condição de forma atenta e assertiva, permitindo, assim, uma abordagem maior do caso. Associado a isso, o encaminhamento ao psiquiatra e a psicoterapia precocemente, como parte da abordagem multidisciplinar e do plano terapêutico (ARCE EPD, et al., 2017).

Como abordado neste estudo, através da análise de um caso clínico, o diagnóstico e o tratamento da SE deve ser conduzido pela dermatologia e acompanhado por uma equipe multiprofissional. Além disso, é importante ressaltar a necessidade de estudos mais aprofundados a respeito da SE, uma vez que há poucos relatos na literatura e o diagnóstico pode estar associado a doenças orgânicas e/ou outras perturbações psiquiátricas.

\section{REFERÊNCIAS}

1. ALVES CJM, et al. Síndrome de Ekbom secundária a transtorno orgânico: relato de três casos. An. Bras. Dermatol., 2010; 85(4): 541-4.

2. AMERICAN PSYCHIATRIC ASSOCIATION. Diagnostic and Statistical Manual of Mental Disorders, Fifth Edition (DSM-5), American Psychiatric Association, Arlington, 2013.

3. AMANCIO EJ, et al. Síndrome de Ekbom e Torcicolo Espasmódico. Arq. Neuro-Psiquiatr., vol.60, no.1, São Paulo, 2002.

4. ARCE EPD, et al. Parasitosis ilusoria intestinal y dermatológica: casos clínicos. Rev. chil. infectol., vol.34, no.3, Santiago, 2017.

5. FREUDENMANN RW, LEPPING P. Delusional infestation. Clin. Microbiol. Rev., 2009; 22 (4): 690-732.

6. GOI PD, SCHARLAU CT. Síndrome de Ekbom acompanhada de Automutilação. Rev. Psiquiatr. RS. 2007; 29(1): 97-99. 
7. JUNIOR QC, CORBETT CEP. Delírio de infestação parasitária e folie à deux: relato de caso. Arq. Neuropsiquiatr., 2003; 61(3B): 872-5.

8. KOO J, LEE CS. Delusions of parasitosis a dermatologist's guide to diagnosis and treatment. Am. J. Clin. Dermatol., p. 2285-90, 2001.

9. LEPPING P, et al. How to approach delusional infestation. BMJ, 2015.

10. LLANOS FC, et al. Psicodermatología en la Atención Primaria. Rev. Hosp. Clín. Univ. Chile, 2016; $27: 27$ - 34.

11. MARQUES JG. Ekbom Syndrome: Case Report and Revision. Lisboa: Centro Hospitalar Psiquiátrico de Lisboa, 2008.

12. MILLER L. An account of insect hallucinations affecting an elderly couple. Canadian Entomology, 1954,86 (10).

13. MUNOZ H, BAYONA L. Síndrome de Ekbom: a propósito de un caso. Rev. Colomb. Psiquiat., 2015; 44(1): 61-65.

14. TRABET W. 100 years of delusions of parasitoses: metanalysis of 1223 case reports. Psychopathology, 1995; 28: 238-246.

15. TERCERO EG, et al. Síndrome de Ekbom: reporte de un caso en un paciente anciano. Rev. Clin. Med. Fam., vol.13, no.1, Albacete feb., 2020.

16. VARGAS CD, et al. Delirio De Parasitosis ou Síndrome De Ekbom. Rev. Méd. La Paz, vol.26, no.1. La Paz, 2020.

17. VIDAL CEL, et al. Delusional parasitosis and bipolar disorder: case report. Rev. Psiquiatr. RS., 2009; 31(1): 79-81. 